\title{
Joint genetic analysis of hippocampal size in mouse and human identifies a novel gene linked to neurodegenerative disease
}

\author{
David G Ashbrook ${ }^{1 *}$, Robert W Williams ${ }^{2}$, Lu Lu ${ }^{2,3}$, Jason L Stein ${ }^{4}$, Derrek P Hibar ${ }^{5}$, Thomas E Nichols ${ }^{6}$,
} Sarah E Medland ${ }^{7}$, Paul M Thompson ${ }^{4}$ and Reinmar Hager ${ }^{1}$

\begin{abstract}
Background: Variation in hippocampal volume has been linked to significant differences in memory, behavior, and cognition among individuals. To identify genetic variants underlying such differences and associated disease phenotypes, multinational consortia such as ENIGMA have used large magnetic resonance imaging (MRI) data sets in human GWAS studies. In addition, mapping studies in mouse model systems have identified genetic variants for brain structure variation with great power. A key challenge is to understand how genetically based differences in brain structure lead to the propensity to develop specific neurological disorders.

Results: We combine the largest human GWAS of brain structure with the largest mammalian model system, the BXD recombinant inbred mouse population, to identify novel genetic targets influencing brain structure variation that are linked to increased risk for neurological disorders. We first use a novel cross-species, comparative analysis using mouse and human genetic data to identify a candidate gene, MGST3, associated with adult hippocampus size in both systems. We then establish the coregulation and function of this gene in a comprehensive systems-analysis.
\end{abstract}

Conclusions: We find that MGST3 is associated with hippocampus size and is linked to a group of neurodegenerative disorders, such as Alzheimer's.

Keywords: Comparative analysis, Hippocampus, MGST3, BXD

\section{Background}

The hippocampus is a key forebrain region involved in declarative memory, cognition, and spatial navigation. Hippocampal volume is highly variable with unilateral values ranging from $\sim 2500$ to $5000 \mathrm{~mm}^{3}$ among healthy young humans (mean $3,917 \mathrm{~mm}^{3}$, s.d. $=441 \mathrm{~mm}^{3}$ ) and from 15.2 to $23.0 \mathrm{~mm}^{3}$ among young adult mice [1,2]. Heritability ranges from $40 \%$ to $70 \%$ in both species $[3,4]$, and a small fraction of the difference in volume is also attributable to sex $[4,5]$. This wide range of natural variation raises the possibility that susceptibility to a subset of neurodegenerative and psychiatric disorders linked to defects in the hippocampus may depend, in part, on its initial healthy volume. Individuals who

\footnotetext{
* Correspondence: david.ashbrook@postgrad.manchester.ac.uk ${ }^{1}$ Computational and Evolutionary Biology, Faculty of Life Sciences, University of Manchester, Michael Smith Building, Oxford Road, Manchester M13 9PT, UK Full list of author information is available at the end of the article
}

develop and retain a large hippocampus into adulthood may be comparatively resistant to some forms of disease, particularly Alzheimer's. Such a "reserve" hypothesis of neurological disease [6,7] has been proposed for Parkinson's [8], Huntington's [9] and Alzheimer's [10] diseases. Lower than average volume has been linked to a number of disorders [11] including depression [12-16], Alzheimer's disease [17] and schizophrenia [18]. Understanding the genetic factors that contribute to individual differences in hippocampal volume is thus crucial in providing insight into vulnerability and severity of disease.

Prior efforts to identify genetic variants underlying differences in brain structure have used large data sets in human genome-wide association studies (GWAS) or extensive mapping populations in mouse model systems. GWAS in humans typically have modest statistical power due to high corrections needed to compensate for multiple testing. However, loci are defined with very high precision, often 
down to the level of single nucleotide polymorphisms (SNPs). In contrast, mouse linkage studies often have high statistical power to detect genetic effects but lower genetic resolution, producing loci that include hundreds of genes $[19,20]$. Combining data from mice and humans overcomes some of these problems, gaining power from mouse crosses and precision from human GWAS. This method also ensures the translational relevance, giving confidence to the human results, as the same gene controlling the same phenotype is found in a related species. Further, this approach illustrates that the homologous mouse gene is relevant to the human phenotype, as well as the significance of experimental research in model systems that would not be possible in humans. Homologous genes are genes that share a common evolutionary ancestor. In this study we are specifically looking at a subset of homologous genes, orthologs, which derive from a speciation event, rather than paralogs, which arise because of a gene duplication event.

This study takes a cross-species approach to identify genes with an evolutionarily conserved role in influencing hippocampus size; i.e. because a given gene is playing the same role in two different species we hypothesize that it was playing the same role in the ancestral species. Previous studies have begun to show the utility of using a crossspecies approach to identify genes underlying a phenotype of interest [21-25]. This approach has the advantage that it allows the investigation of disease phenotypes without requiring data from experimental perturbations. Instead we utilize data obtained from populations that segregated for large numbers of common sequence variants and associated differences in phenotype.

Here, we use data from the largest mouse model system, BXD, to identify a set of genes associated with hippocampus size in a joint analysis with human hippocampus MRI data obtained by the ENIGMA consortium for GWAS [26]. We identify, MGST3 [Entrez: 4259] and use a systems-genetics approach that links this gene to neurodegenerative disorders such as Alzheimer's disease and Parkinson's disease.

\section{Results}

\section{Identification of genes significant in both species}

Associations between genes and hippocampus size in BXD mice were estimated using p-values for over 3800 markers obtained for QTL interval mapping. QTL mapping identifies a region of the genome significantly linked to variation in the phenotype. Having identified QTL, we then estimated a particular gene's significance based on its base pair distance from the nearest two markers and the significance of these two markers. Therefore any particular gene will have a $\mathrm{p}$-value somewhere between the p-values of its two closest markers. The next step in our analysis was to obtain SNP level pvalues for association with human hippocampus volume, which were converted to gene p-values to allow comparison with data for the mouse hippocampus. Using the SNP p-values from the human GWAS, the Versatile Gene-based Association Study (VEGAS) website [27] produced gene p-values for a total of 17,787 human genes. Secondly, the mouse homologues of these human genes were identified and yielded a total of 15,705 genes $(88.3 \%$ of the human genes).

Using a relaxed (i.e. uncorrected) p-value of $\leq 0.05$, 1015 human genes (916 with mouse homologues; 90.2\%) were then nominally identified as having an effect on hippocampus size. Overall, there is no indication that the significance of any given gene with the entire region identified in the QTL analysis of BXD mouse hippocampus weight is indicative of the homologous gene's significance on human hippocampus volume, as judged by the quantile-quantile plot and lambda $(\lambda=0.912, p=0.82$; Figure 1). This is corroborated by a separate Rank Rank Hypergeometric Overlap test [28] used to compare the two datasets, which yielded a non-significant result $(\mathrm{p}=$ 0.38 , corrected by the familywise error rate). This is unsurprising as a QTL analysis identifies a region of the genome associated with a trait, and therefore in our analysis all genes within the mouse QTL were significant. However, not all the genes within a QTL contribute to the phenotype, but only a subset or even a single gene.

Therefore we sought to identify which genes are associated with both BXD mouse hippocampus weight and human hippocampus volume. The 42 genes which were significant in mouse are all within a QTL on chromosome 1 [4,29]. Only one gene had a significant human p-value

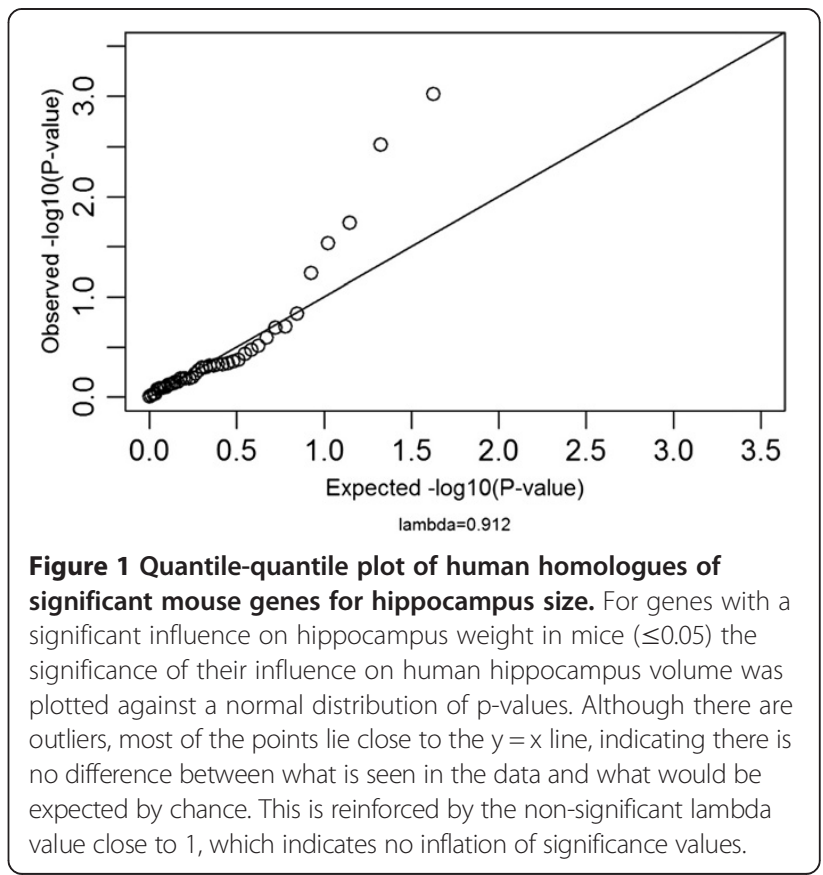


when corrected for multiple testing $(0.05 / 42=\mathrm{p} \leq 0.0012$; Additional file 1), MGST3.

\section{Regulation of gene expression}

To establish if our candidate gene was regulating its own expression, mouse hippocampus microarray data from GeneNetwork was used to find probes corresponding to expression of the gene, and WebQTL was used to produce eQTL [30]. Of the 17 probes for Mgst3 [Entrez: 66447] within the exon array data, six have a significant Pearson's correlation $(r \geq 0.5, p \leq 0.05)$, and these probes were used to represent expression of Mgst 3 in the mouse hippocampus. The six probes represent all four probes for exons and one each from the 5' and 3' UTR (Table 1). The remaining 11 probes were for introns and UTRs. This shows that the correlating probes represent the protein coding parts of the gene.

Mgst3 has a cis-eQTL, suggesting it regulates its own expression. No trans-QTL was found which was consistent between probes. The QTL and eQTL analysis also showed that the C57BL/6 J (B6) allele increased hippocampus weight, whereas the DBA/2 J (D2) allele increased the expression of Mgst3.

\section{Functional analysis of significant genes}

To investigate the function of our candidate genes, we used the Database for Annotation, Visualization and Integrated Discovery (DAVID) as it allows us to analyse a number of different annotation databases. Significance was determined by the false discovery rate (FDR), which corrects the significance value for the large number of multiple comparisons.

To determine if any annotations were enriched in both mouse and human, even though individual genes were not shared, separate lists of genes nominally significant in human (915 genes $\mathrm{p} \leq 0.05)$ and human homologues of the 42 mouse genes with $\mathrm{p} \leq 0.05$ were entered into DAVID. No overlapping significant annotations were found, i.e. no annotations were significantly enriched in both the genes significant in human and the genes significant in mouse. Again, this shows that not all 42 genes within the mouse QTL influence the phenotype, but only a subset.

\section{Guilt-by-association}

Coexpression of genes implies that they share the same regulatory mechanisms [31] and/or are involved in the same biological processes. A 'guilt-by-association' approach asserts that the function of a gene, or list of genes, can be indicated by the genes that it commonly coexpresses with, as common coexpression indicates they are part of the same biological process [32]. The large datasets of gene expression provided by GeneNetwork and GeneFriends allows this 'guilt-by-association' approach to be used. This is especially useful for genes such as MGST3/Mgst3, which previously have not been investigated in detail.

GeneFriends shows human genes which coexpress in a large number of datasets from the Gene Expression omnibus. However it is not specific for tissue or treatment. This identified 8135 genes that were coexpressed with MGST3 in over half of the datasets (coexpression value $\geq 0.5$; Additional file 2). These were analysed using DAVID, to find what KEGG pathway annotations were significant (FDR $\leq 0.05$; Additional file 3 ). One of the six KEGG pathways is particularly interesting; Alzheimer's disease (FDR $=0.0029)$.

To support a specific link between genes that are coexpressed with Mgst3 and Alzheimer's disease we used the exon array data from GeneNetwork, as this is specific to the hippocampus. Each of the six above identified probes for Mgst3 was correlated against the entire exon array

Table 1 Pearson correlations between probes for Mgst3 in adult mouse hippocampus

\begin{tabular}{|c|c|c|c|c|c|c|c|c|c|}
\hline & Probe ID & Location (Mbp) & Target & 1 & 2 & 3 & 4 & 5 & 6 \\
\hline \multirow[t]{2}{*}{1} & 4654447 & 169.302579 & 3'UTR & 1 & $r=0.921$ & $r=0.873$ & $r=0.787$ & $r=0.8$ & $r=0.62$ \\
\hline & & & & & $p<1 E-16$ & $p<1 E-16$ & $p<1 E-16$ & $p<1 E-16$ & $p=9.87 E-10$ \\
\hline \multirow[t]{2}{*}{2} & 5358488 & 169.302648 & exon5 & $r=0.921$ & 1 & $r=0.903$ & $r=0.84$ & $r=0.828$ & $r=0.632$ \\
\hline & & & & $p<1 E-16$ & & $p<1 E-16$ & $p<1 E-16$ & $p<1 E-16$ & $p=3.71 E-10$ \\
\hline \multirow[t]{2}{*}{3} & 5399827 & 169.303925 & exon4 & $r=0.873$ & $r=0.903$ & 1 & $r=0.937$ & $r=0.884$ & $r=0.534$ \\
\hline & & & & $p<1 \mathrm{E}-16$ & $p<1 E-16$ & & $p<1 E-16$ & $p<1 E-16$ & $p=5.33 E-7$ \\
\hline \multirow[t]{2}{*}{4} & 5566068 & 169.307412 & exon2 & $r=0.787$ & $r=0.84$ & $r=0.937$ & 1 & $r=0.92$ & $r=0.512$ \\
\hline & & & & $p<1 E-16$ & $p<1 E-16$ & $p<1 E-16$ & & $p<1 E-16$ & $p=1.84 E-6$ \\
\hline \multirow[t]{2}{*}{5} & 5025657 & 169.308444 & exon1 & $r=0.8$ & $r=0.828$ & $r=0.884$ & $r=0.92$ & 1 & $r=0.519$ \\
\hline & & & & $p<1 E-16$ & $p<1 E-16$ & $p<1 E-16$ & $p<1 E-16$ & & $p=1.24 E-6$ \\
\hline \multirow[t]{2}{*}{6} & 5280988 & 169.323882 & $5^{\prime}$ UTR & $r=0.62$ & $r=0.632$ & $r=0.534$ & $r=0.512$ & $r=0.519$ & 1 \\
\hline & & & & $p=9.87 E-10$ & $p=3.71 E-10$ & $p=5.33 E-7$ & $\mathrm{p}=1.84 \mathrm{E}-6$ & $p=1.24 \mathrm{E}-6$ & \\
\hline
\end{tabular}


dataset (1,236,087 probes) to find the top 20,000 probes with which it correlates. These six lists of probes were then combined to find which probes correlated with all six probes for Mgst3. This produced a list of 5906 probes which correlated with all six of the probes for Mgst3, representing 2971 genes (Additional file 4). Permutation testing was carried out to determine an empirical p-value for how often six lists of 20,000 values from a choice of $1,236,087$ values would overlap, and produced a $\mathrm{p}$-value of $<1 \times 10^{-6}$. This shows that the overlap between our probes is highly significant, and that these 2971 genes really do coexpress with Mgst3 in the mouse hippocampus. Enrichment of this list of genes was then examined in DAVID, and again we see genes involved in neurodegenerative diseases significantly enriched (Additional file 5): Huntington's disease (95 genes, FDR $=3.29 \mathrm{E}-27$, Parkinson's disease (77 genes, FDR $=1.56 \mathrm{E}-25)$ and Alzheimer's disease (83 genes, FDR $=1.29 \mathrm{E}-18$ ).

Finally, the overlap between the genes that are commonly coexpressed with MGST3 and human homologues of the genes that are coexpressed with Mgst3 in the mouse hippocampus was examined. This showed that 1579 genes which commonly coexpress with MGST3 also coexpress with its mouse homologue in the mouse hippocampus (Additional file 6). We tested this by permutation taking samples of 8135 genes and 2971 genes from a list of all known human protein coding genes and determining how often an overlap larger than 1579 was seen. This produced an empirical p-value $\leq 1 \times 10^{-6}$. Again with KEGG enrichment analysis, the three neurodegenerative diseases are highly significant (Additional file 7): Huntington's disease (78 genes, FDR $=3.08 \mathrm{E}-22$ ), Parkinson's disease (63 genes, FDR $=5.68 \mathrm{E}-21)$ and Alzheimer's disease (69 genes $(\mathrm{FDR}=1.34 \mathrm{E}-18)$.

\section{Discussion}

We found strong evidence that MGST3, on chromosome 1 in both mouse and human, is significantly associated with hippocampus size. MGST3 has previously been found to be down-regulated in Alzheimer's disease [33]. The fact that the gene appears to have an evolutionarily conserved role in both species suggests a role in hippocampus morphology. MGST3 has been found to be particularly highly expressed in the rat hippocampus [34]. A 'guilt-by-association' approach shows that these genes coexpress with genes linked to neurodegenerative disorders associated with reduced hippocampus volume: Huntington's disease [35-37], Alzheimer's disease [17,38] and Parkinson's disease [39-41].

The potential mechanism for this link is more speculative. Genes that coexpress with MGST3 are also associated with cellular energy production, as the oxidative phosphorylation KEGG pathway appears in our results [Additional files 3, 5 and 7]. Mitochondrial dysfunction has been implicated in both neuropsychiatric and neurodegenerative disorders $[42,43]$, linking the mitochondrial and neurodegenerative annotations. Recently it has been reported that dysfunction of mtDNA genes, which have been implicated in Alzheimer's disease, directly influence left hippocampal atrophy [44]. Further, links have also been found between oxidative stress and regulation of Mgst3 in mice [45].

MGST3 has also been linked to inflammation, as it and other family members show leukotriene C4 ( $\left.\mathrm{LTC}_{4}\right)$ synthase activity. Leukotrienes are physiological important mediators of various inflammatory and immediate hypersensitivity processes [46]. When porcine kidney cells were exposed a nephrotoxin, aristolochic acid I, MGST3 and FLAP (another family member) were upregulated before an increase in LTs synthesis. This is relevant as Alzheimer's disease, as well as other neurodegenerative disorders, have been linked to inflammation (reviewed in [47]). However, other research has found that rat MGST3 does not have $\mathrm{LTC}_{4}$ synthase activity [48], is not upregulated in response to lipopolysaccharide [49] and does not appear to be directly involved in the inflammation response [34]. In this last paper, the authors speculate that it may have a neuroprotective role against oxidative stress [34].

\section{Conclusions}

In summary, the combination of human GWAS and mouse QTL data from some of the largest study systems available has enabled us to identify a novel gene, MGST3, which is associated with hippocampus size across species and, when dysregulated, is linked to neurodegenerative disorders.

\section{Methods}

\section{Data}

We used mouse hippocampus weight data from 35 BXD lines plus the parental C57BL/6 J and DBA/2 J strains, adjusted for age, sex, body weight, and brain weight minus hippocampus weight $[4,30]$ (GN13031). Over 3800 SNP markers are used for QTL interval mapping, thus for each marker significance values are available. Using the gene's base pair distance from the nearest two markers we developed a python script [50] to produce an estimated p-value for each gene. For example a gene positioned halfway between two markers would have an estimated p-value half way between the two marker values. Therefore an estimated $\mathrm{p}$-value could be produced for any gene in the mouse genome (NCBI37/mm9) by using the gene's known start position and any set of mouse markers.

Human MRI-generated hippocampus volume from healthy subjects and patients was generated for GWAS meta-analyses by the Enhancing Neuro Imaging Genetics Through Meta-Analysis (ENIGMA) network [1,26,51] and 
can be visualised with ENIGMAvis [51]. Association analyses used multiple linear regression with hippocampus volume as a dependent variable and the additive dosage of each SNP as an independent variable, controlling for covariates of population stratification (four MDS components), intracranial volume, age, age ${ }^{2}$, sex and the interactions between age and sex and age ${ }^{2}$ and sex. Dummy covariates were used to control for different scanner sequences or equipment within a site. We converted the p-values available for each SNP marker to gene level significance values using the Versatile Genebased Association Study [27] website (VEGAS). This tool tests for association between the phenotype and a gene by summarizing the full set of SNPs in the gene. Linkage disequilibrium between SNPs in a gene is taken into account by using simulation based on the precalculated linkage disequilibrium structure of a set of reference individuals from the HapMap phase $2 \mathrm{CEU}$ population. SNPs are assigned to each of 17,787 autosomal genes on the UCSC Genome Browser hg18 assembly, with boundaries defined as $\pm 50 \mathrm{~kb}$ of $5^{\prime}$ and $3^{\prime}$ UTRs. Association p-values for any given gene with $n$ SNPs are converted to uppertail chi-squared statistics with one degree of freedom (df). The gene-based test statistic is then the sum of all of the chi-squared $1 \mathrm{df}$ statistics within that gene. If the SNPs are in perfect linkage equilibrium, the test statistic will have a chisquared distribution with $\mathrm{n}$ degrees of freedom under the null hypothesis. However this is unlikely to be the case, therefore the true null distribution given the LD structure (and hence p-values that correlate accordingly) will need to be taken into account. This is done by simulating a large number of multivariate normal vectors, and the empirical gene based p-value is the proportion of simulated test statistics that exceed the observed gene-based test statistic [27]. Thus, we are able to identify genes associated with hippocampus size that may be significant, independent of whether individual SNPs are significant.

All data used for the above is from existing, previously published, anonymised data and therefore no further ethical approval was needed.

\section{Identification of significant genes for hippocampus size in mouse and human}

To be able to compare the data between species, mouse homologues for the human genes need to be identified. The marker method above can produce a p-value for any mouse gene, therefore it is the human genes produced by VEGAS that limit the total number of genes in our analysis. Using the human SNP p-values, VEGAS produced pvalues for 17,787 human genes (the number of autosomal genes on the UCSC Genome Browser hg18 assembly). We used several tools to identify mouse homologues for these human genes: MammalHom [52], Mouse Genome
Informatics [53] and HomoloGene [54]. Thus, 15,705 mouse genes with a corresponding human homologue were identified, representing $88.3 \%$ of human genes.

To determine if genes affecting hippocampus weight in mouse also influence hippocampus volume in humans we used a protocol developed in R [55]. Firstly, we produced a quantile-quantile plot using the human p-values of those of the 42 genes which had a mouse $p$-value of $\leq 0.05$. Secondly, the genomic control $\lambda$-value [56] was calculated. This value is generally a measure of inflation of statistics due to population stratification, i.e. if significance is increased due to the populations being related. In our case a high lambda would show that overall those genes with a significant mouse p-value have a higher human $\mathrm{p}$-value than would be expected by chance. In other words, by using genes which are significant in mouse, the p-values of the homologous human genes would be inflated. In our study we tested this value by permutations, with the same number of random genes sampled from the genome and the $\lambda$-value calculated (random $\lambda$ ). The number of times that the random $\lambda$ was greater than the calculated $\lambda$ was divided by the number of permutations $(100,000)$ to give the p-value of the calculated lambda values. The permutations determine if a high $\lambda$-value is simply due to an overall high $\lambda$ between the two datasets, i.e. that all the p-values in human are higher than would be expected by chance. We validated results thus obtained using an additional approach, the Rank Rank Hypergeometric Overlap test [28]. This was carried out using the RRHO R package [57], which computes the number of overlapping elements, and return the observed significance of this overlap using a hypergeometric test.

Thirdly, to assess if any particular gene is associated with brain region size in both mouse and human the significance of the homologues for the 42 genes found to be significant in BXD mice were examined in the human GWAS data. This was corrected for multiple comparisons using the number of genes compared [23] (42 significant mouse genes), therefore $0.05 / 42=\mathrm{p}<0.0012$.

\section{Expression quantitative trait loci}

Expression quantitative trait loci (eQTL) show regions of the genome that influence the expression of a gene of interest. A cis-eQTL, i.e. an eQTL in the same position of the candidate gene, suggests that the candidate gene regulates its own expression, whereas a trans-eQTL, i.e. a QTL elsewhere in the genome, indicates that a gene at this position is influencing the expression of the candidate gene. Data for exon mRNA expression in the hippocampus of mouse lines (mainly BXD but with data from other inbred mouse lines) available at GeneNetwork were used and WebQTL [58] produced eQTL for genes identified above [30]. The database of microarray results used from GeneNetwork was UMUTAffy Hippocampus Exon 
(Feb09) RMA (GN206) [59]. This allows the examination of the cis- or trans- regulation of our identified gene (Mgst3) in the mouse hippocampus. Using this exon gene expression data, all probes for Mgst3 were correlated using Pearson's product-moment correlation as implemented in GeneNetwork, and those probes which showed a significant correlation $(r \geq 0.5, p \leq 0.05)$ were said to represent the expression of the gene.

\section{Functional analysis}

Functional analysis allows us to investigate enrichment; for example if the molecular function of a gene product is over-represented in a submitted list of genes. Enrichment therefore suggests whether a particular gene or a set of genes is associated with a particular function or disorder.

The Database for Annotation, Visualization and Integrated Discovery (DAVID) $[60,61]$ identifies if a given list of genes is significantly enriched in an annotated gene term. DAVID uses a range of databases, including Gene Ontologies (GO) terms [62], Kyoto Encyclopedia of Genes and Genomes (KEGG) pathways [63], Online Mendelian Inheritance in Man diseases and InterPro protein domains [64]. Separate lists of all significant mouse genes $(\mathrm{p} \leq 0.05)$ and all nominally significant human genes $(\mathrm{p} \leq 0.05)$ were analysed, and the results examined for any annotations that appeared in both datasets. The latter would suggest that the same pathways or networks were involved in the phenotype in both species, even if the same individual genes are not significant.

\section{Coexpresion and 'Guilt-by-association'}

Shared regulation and function of genes can also be established using coexpression analysis [31]. However, coexpression can differ between species or between tissues within an organism. To examine if genes are commonly coexpressed in humans, GeneFriends can be used [32]. GeneFriends takes submitted list of genes and uses a large database of microarray data (4164 Micro array datasets containing 26,113 experimental conditions and 19,080 genes) [32], from the Gene Expression Omnibus [65,66] to find genes that are commonly coexpressed with the entered gene list. However it is not specific for tissue type or treatment, and therefore can only inform us which genes tend to coexpress together, and not which genes specifically coexpress in the hippocampus or at what time points.

GeneFriends produces a list of genes that are coexpressed with the submitted genes in a significant number of datasets, to identify commonly coexpressed genes (coexpressed independent of treatment or tissue). Common coexpression suggests that the genes are under the same regulation in particular since coexpression is analysed across conditions and tissues. This list of commonly coexpressed genes was analysed using DAVID as above, producing annotations for these genes. This allows a 'guilt-by-association' approach, where the roles played by genes that are commonly coexpressed with our genes are used to suggest the networks that the genes are part of [32]. We next used Pearson productmoment correlations, as implemented in GeneNetwork, to examine coexpression in mice by producing correlation matrices of hippocampal gene expression [67]. In contrast to GeneFriends this is specific to the hippocampus. Hippocampus mRNA expression was found for Mgst3 in the UMUTAffy Hippocampus Exon (Feb09) RMA (GN206) microarray database. The probes for Mgst3 were correlated with each other, and six showed a significant correlation $(\mathrm{r} \geq 0.5, \mathrm{p} \leq 0.05)$ and were used to determine gene expression. For each of these six correlating probes for Mgst3, the top 20,000 correlations were then found within the whole hippocampus exon array dataset (1,236,087 probes). 5906 probes correlated with all six of the probes for Mgst3, representing 2971 genes. This list of 2971 was submitted to DAVID to determine KEGG pathway enrichment. Significance testing using permutations was then carried out to determine the overlap between six random samples of 20,000 values (the number of correlations) from a total of 1,236,087 values (the total number of probes). With $1,000,000$ permutations this produced a value of $\mathrm{p}<1 \times 10^{-6}$.

The overlap between the genes identified by GeneFriends and those identified by GeneNetwork was also examined. The resulting list of genes was then submitted to DAVID for KEGG pathway enrichment analysis. The significance of the number of overlapping genes was again determined by permutation. Samples of sizes 8135 (the number of coexpressing genes found by GeneFriends) and of 2971 (the number of coexpressing genes found by GeneNetwork) were taken from a list of all protein coding human genes (downloaded from the HUGO gene nomenclature committee website $[68,69])$, and the overlap between these two samples recorded. This was repeated $1,000,000$ times and the significance value was calculated by dividing the number of times the overlap between the two samples was greater than 1579 (the overlap we see) by the number of permutations $(1,000,000)$.

\section{Additional files}

Additional file 1: Genes with significant $p$-value $(p \leq 0.05)$ associated with hippocampus weight in BXD. A table showing the human homologue gene symbols for all the mouse genes with a genome wide $p$-value $\leq 0.05$ for hippocampus weight in BXD, showing their unadjusted p-value in human.

Additional file 2: Genes which are commonly coexpressed with MGST3 as determined by GeneFriends. A table showing the genes which are commonly coexpressed with MGST3, independent of tissue or treatment, as identified by GeneFriends. For each gene its Entrez gene ID, gene symbol and coexpression value with MGST3 are shown. 
Additional file 3: KEGG pathway annotations enriched in genes that are coexpressed with MGST3. A table showing KEGG pathway annotations significantly enriched (calculated by DAVID) in genes that are significantly coexpressed with MGST3 (calculated by GeneFriends). The table shows the category of the enrichment and the specific annotation, the number of submitted genes in the annotation/total number of submitted genes in the category, the fold enrichment and the FDR.

Additional file 4: Probes which correlate with all six of the correlating probes for Mgst 3 in the adult mouse hippocampus as determined by Pearson correlations in GeneNetwork. A table showing the probes which correlate with all six of the Mgst3 probes which correlate together. For each probe, the probe ID, Entrez gene ID of the mouse gene, Entrez gene ID of the homologous gene, the mouse gene symbol and the gene's location is given.

\section{Additional file 5: KEGG pathway annotations enriched in genes} that are coexpressed with Mgst3 in the adult mouse hippocampus. A table showing KEGG pathway annotations significantly enriched (calculated by DAVID) in genes significantly coexpressed with Mgst3 (calculated by Pearson correlation in GeneFriends). The table shows the category of the enrichment and the specific annotation, the number of submitted genes in the annotation/total number of submitted genes in the category, the fold enrichment and the FDR.

Additional file 6: Genes which commonly coexpress with MGST3 as determined by GeneFriends and which coexpress with Mgst 3 in the adult mouse. A table showing the homologous genes which commonly coexpress with MGST3, independent of tissue or treatment (as identified by GeneFriends) and those which are coexpressed with Mgst3 in the adult mouse hippocampus, as determined by Pearson correlation in GeneNetwork. For each gene, its Human gene symbol, Human Entrez gene ID, Human chromosome, Human gene location, Mouse Gene Symbol, Mouse Entrez Gene ID, Mouse chromosome and Mouse gene location is shown.

Additional file 7: KEGG pathway annotations enriched in genes which are coexpressed with MGST3 (as determined by GeneFriends) and those which coexpress with Mgst3 in the adult mouse. A table showing the homologous genes which commonly coexpress with MGST3, independent of tissue or treatment (as identified by GeneFriends) and which coexpress with Mgst 3 in the adult mouse hippocampus, as determined by Pearson correlation in GeneNetwork. The table shows the category of the enrichment and the specific annotation, the number of submitted genes in the annotation/total number of submitted genes in the category, the fold enrichment and the FDR.

\section{Abbreviations}

BXD: A large set of recombinant inbred strains derived by crossing C57BL/6 J and DBA/2 I and inbreeding progeny for 20 or more generations.; CEU: Utah residents with Northern and Western European ancestry; DAVID: Database for annotation, visualization and integrated discovery; Df: Degree of freedom; ENIGMA: Enhancing neuro imaging genetics through meta-analysis; eQTL: Expression quantitative trait loci; FDR: False discovery rate; GO: Gene ontology; GWAS: Genome wide association study; HUGO: Human genome organisation; KEGG: Kyoto encyclopedia of genes and genomes; LD: Linkage disequilibrium; LRS: Likelihood ratio statistic; MGl: Mouse genome informatics; MGST3: Microsomal glutathione S-transferase 3 [Entrez 4259]; Mgst3: Microsomal glutathione S-transferase 3 [Entrez 66447]; MRI: Magnetic resonance imaging; mRNA: Messenger ribonucleic acid; QTL: Quantitative trait loci; SNP: Single nucleotide polymorphism; UCSC: University of California, Santa Cruz; UTR: Untranslated region; VEGAS: Versatile gene-based association study.

\section{Competing interests}

The authors declare that they have no competing interests.

\section{Authors' contributions}

DGA, RWW, LL, DPH, JS, PT and RH designed research; DGA, RWW, LL, DPH, JS and RH performed research. DGA, RWW, LL, DPH, JS, and RH analyzed data. TEN, SEM, contributed reagents. DGA, RWW, JS, PT and RH wrote the paper. All authors read and approved the final manuscript.

\section{Acknowledgements}

We would like to thank the ENIGMA consortium members for the use of the human hippocampus data and help with the comparative analysis. DGA is supported by a BBSRC studentship and The University of Manchester. LL's work is supported by funding from NIH (R01 EY021200) and National Natural Science Foundation of China (Grant No. 30971591). GeneNetwork is supported by NIAAA Integrative Neuroscience Initiative on Alcoholism (U01 AA016662, U01 AA013499) NIDA, NIMH, and NIAAA (P20-DA 21131) and the UTHSC Center for Integrative and Translational Genomics. JLS was funded by the Autism Speaks Translational Postdoctoral Fellowship.

\section{Author details}

'Computational and Evolutionary Biology, Faculty of Life Sciences, University of Manchester, Michael Smith Building, Oxford Road, Manchester M13 9PT, UK. 2University of Tennessee Health Science Center, Memphis, TN 38163, USA. ${ }^{3}$ Jiangsu Key Laboratory of Neuroregeneration, Nantong University, Nantong, China. ${ }^{4}$ Department of Neurology, Laboratory of Neuro Imaging, UCLA School of Medicine, Los Angeles, CA 90095-1769, USA. Institute for Neuroimaging and Informatics, Imaging Genetics Center, Keck School of Medicine, University of Southern California, 2001 N. Soto Street, Los Angeles, CA 90033, USA.

${ }^{6}$ Department of Statistics \& Warwick Manufacturing Group, The University of Warwick, Coventry CV4 7AL, UK. ${ }^{7}$ Genetic Epidemiology Laboratory, Queensland Institute of Medical Research Berghofer, Brisbane, Australia.

Received: 7 April 2014 Accepted: 29 September 2014

Published: 3 October 2014

\section{References}

1. Stein JL, Medland SE, Vasquez AA, Hibar DP, Senstad RE, Winkler AM, Toro R, Appel K, Bartecek R, Bergmann $\varnothing$, Bernard M, Brown AA, Cannon DM, Chakravarty MM, Christoforou A, Domin M, Grimm O, Hollinshead M, Holmes AJ, Homuth G, Hottenga J-J, Langan C, Lopez LM, Hansell NK, Hwang KS, Kim S, Laje G, Lee PH, Liu X, Loth E, et al: Identification of common variants associated with human hippocampal and intracranial volumes. Nat Genet 2012, 44:552-561.

2. Peirce JL, Chesler EJ, Williams RW, Lu L: Genetic architecture of the mouse hippocampus: identification of gene loci with selective regional effects. Genes Brain Behav 2003, 2:238-252.

3. Peper JS, Brouwer RM, Boomsma DI, Kahn RS, Hulshoff Pol HE: Genetic influences on human brain structure: a review of brain imaging studies in twins. Hum Brain Mapp 2007, 28:464-473.

4. Lu L, Airey DC, Williams RW: Complex trait analysis of the hippocampus: mapping and biometric analysis of two novel gene loci with specific effects on hippocampal structure in mice. J Neurosci 2001, 21:3503-3514.

5. Fjell AM, Westlye LT, Amlien I, Espeseth T, Reinvang I, Raz N, Agartz I, Salat DH, Greve DN, Fischl B, Dale AM, Walhovd KB: Minute effects of sex on the aging brain: a multisample magnetic resonance imaging study of healthy aging and Alzheimer's disease. J Neurosci 2009, 29:8774-8783.

6. Barnett JH, Salmond CH, Jones PB, Sahakian BJ: Cognitive reserve in neuropsychiatry. Psychol Med 2006, 36:1053-1064.

7. Nithianantharajah J, Hannan AJ: The neurobiology of brain and cognitive reserve: mental and physical activity as modulators of brain disorders. Prog Neurobiol 2009, 89:369-382.

8. Biundo R, Calabrese M, Weis L, Facchini S, Ricchieri G, Gallo P, Antonini A: Anatomical correlates of cognitive functions in early Parkinson's disease patients. PLoS One 2013, 8:e64222.

9. Bonner-Jackson A, Long JD, Westervelt $\mathrm{H}$, Tremont $\mathrm{G}$, Aylward E, Paulsen JS: Cognitive reserve and brain reserve in prodromal Huntington's Disease. J Int Neuropsychol Soc 2013, 19:739-750.

10. Braskie MN, Thompson PM: Understanding cognitive deficits in Alzheimer's disease based on neuroimaging findings. Trends Cogn Sci 2013, 17:510-516.

11. Geuze E, Vermetten E, Bremner JD: MR-based in vivo hippocampal volumetrics: 2. Findings in neuropsychiatric disorders. Mol Psychiatry 2005, 10:160-184.

12. Frodl T, Meisenzahl EM, Zetzsche T, Born C, Groll C, Jäger M, Leinsinger $G$, Bottlender R, Hahn K, Möller H-J: Hippocampal changes in patients with a first episode of major depression. Am J Psychiatry 2002, 159:1112-1118.

13. Videbech P, Ravnkilde B, Ph D: Hippocampal volume and depression: a meta-analysis of MRI studies. Am J Psychiatry 2004, 161:1957-1966. 
14. Sheline YI: Depression and the hippocampus: cause or effect? Biol Psychiatry 2011, 70:308-309.

15. Sexton CE, Le Masurier M, Allan CL, Jenkinson M, McDermott L, Kalu UG, Herrmann LL, Bradley KM, Mackay CE, Ebmeier KP: Magnetic resonance imaging in late-life depression: vascular and glucocorticoid cascade hypotheses. Br J Psychiatry 2012, 201:46-51.

16. Sawyer K, Corsentino E, Sachs-Ericsson N, Steffens DC: Depression, hippocampal volume changes, and cognitive decline in a clinical sample of older depressed outpatients and non-depressed controls. Aging Ment Health 2012, 16:753-762.

17. Den Heijer T, Geerlings MI, Hoebeek FE, Hofman A, Koudstaal PJ, Breteler MMB: Use of hippocampal and amygdalar volumes on magnetic resonance imaging to predict dementia in cognitively intact elderly people. Arch Gen Psychiatry 2006, 63:57-62

18. Adriano F, Caltagirone C, Spalletta G: Hippocampal volume reduction in first-episode and chronic schizophrenia: a review and meta-analysis. Neuroscientist 2012, 18:180-200.

19. Mackay TFC, Stone EA, Ayroles JF: The genetics of quantitative traits: challenges and prospects. Nat Rev Genet 2009, 10:565-577.

20. Ackert-Bicknell CL, Karasik D, Li Q, Smith RV, Hsu Y-H, Churchill GA, Paigen BJ, Tsaih S-W: Mouse BMD quantitative trait loci show improved concordance with human genome-wide association loci when recalculated on a new, common mouse genetic map. J Bone Miner Res 2010, 25:1808-1820.

21. Poot M, Badea A, Williams RW, Kas MJ: Identifying human disease genes through cross-species gene mapping of evolutionary conserved processes. PLoS One 2011, 6:e18612.

22. Koutnikova H, Laakso M, Lu L, Combe R, Paananen J, Kuulasmaa T, Kuusisto J, Häring H-U, Hansen T, Pedersen O, Smith U, Hanefeld M, Williams RW, Auwerx J: Identification of the UBP1 locus as a critical blood pressure determinant using a combination of mouse and human genetics. PLoS Genet 2009, 5:e1000591

23. De Mooij-van Malsen AJG, van Lith HA, Oppelaar H, Hendriks J, de Wit M, Kostrzewa E, Breen G, Collier DA, Olivier B, Kas MJ: Interspecies trait genetics reveals association of Adcy8 with mouse avoidance behavior and a human mood disorder. Biol Psychiatry 2009, 66:1123-1130.

24. Leduc MS, Lyons M, Darvishi K, Walsh K, Sheehan S, Amend S, Cox A Orho-Melander M, Kathiresan S, Paigen B, Korstanje R: The mouse QTL map helps interpret human genome-wide association studies for HDL cholesterol. J Lipid Res 2011, 52:1139-1149.

25. Schofield PN, Hoehndorf R, Gkoutos GV: Mouse genetic and phenotypic resources for human genetics. Hum Mutat 2012, 33:826-836.

26. Thompson PM, Stein JL, Medland SE, Hibar DP, Vasquez AA, Renteria ME, Toro R, Jahanshad N, Schumann G, Franke B, Wright MJ, Martin NG, Agartz I, Alda M, Alhusaini S, Almasy L, Almeida J, Alpert K, Andreasen NC, Andreassen OA, Apostolova LG, Appel K, Armstrong NJ, Aribisala B, Bastin ME, Bauer M, Bearden CE, Bergmann O, Binder EB, Blangero J, et al: The ENIGMA consortium: large-scale collaborative analyses of neuroimaging and genetic data. Brain Imaging Behav 2014, In Press.

27. Liu JZ, McRae AF, Nyholt DR, Medland SE, Wray NR, Brown KM, Hayward NK, Montgomery GW, Visscher PM, Martin NG, Macgregor S: A versatile gene-based test for genome-wide association studies. Am J Hum Genet 2010, 87:139-145.

28. Plaisier SB, Taschereau R, Wong JA, Graeber TG: Rank-rank hypergeometric overlap: identification of statistically significant overlap between gene-expression signatures. Nucleic Acids Res 2010, 38:e169.

29. Hager R, Lu L, Rosen GD, Williams RW: Genetic architecture supports mosaic brain evolution and independent brain-body size regulation. Nat Commun 2012, 3(May):1079.

30. GeneNetwork; http://www.genenetwork.org/.

31. Allocco DJ, Kohane IS, Butte AJ: Quantifying the relationship between co-expression, co-regulation and gene function. BMC Bioinformatics 2004, 5:18.

32. Van Dam S, Cordeiro R, Craig T, van Dam J, Wood SH, de Magalhães JP: GeneFriends: an online co-expression analysis tool to identify novel gene targets for aging and complex diseases. BMC Genomics 2012, 13:535.

33. Xu P-T, Li Y-J, Qin X-J, Scherzer CR, Xu H, Schmechel DE, Hulette CM, Ervin J, Gullans SR, Haines J, Pericak-Vance MA, Gilbert JR: Differences in apolipoprotein $\mathrm{E} 3 / 3$ and E4/4 allele-specific gene expression in hippocampus in Alzheimer disease. Neurobiol Dis 2006, 21:256-575.

34. Fetissov SO, Schröder O, Jakobsson P-JP-JP-J, Samuelsson B, Haeggström JZ, Hökfelt T: Expression of microsomal glutathione S-transferase type 3 mRNA in the rat nervous system. Neuroscience 2002, 115:891-897.
35. Spargo E, Everall IP, Lantos PL: Neuronal loss in the hippocampus in Huntington's disease: a comparison with HIV infection. J Neurol Neurosurg Psychiatry 1993, 56:487-491.

36. Bohanna I, Georgiou-Karistianis N, Hannan AJ, Egan GF: Magnetic resonance imaging as an approach towards identifying neuropathological biomarkers for Huntington's disease. Brain Res Rev 2008, 58:209-225.

37. Rosas HD, Koroshetz WJ, Chen Yl, Skeuse C, Vangel M, Cudkowicz ME, Caplan K, Marek K, Seidman LJ, Makris N, Jenkins BG, Goldstein JM: Evidence for more widespread cerebral pathology in early HD: an MRI-based morphometric analysis. Neurology 2003, 60:1615-1620.

38. Frisoni GB, Fox NC, Jack CR, Scheltens P, Thompson PM: The clinical use of structural MRI in Alzheimer disease. Nat Rev Neurol 2010, 6:67-77.

39. Camicioli R, Moore MM, Kinney A, Corbridge E, Glassberg K, Kaye JA: Parkinson's disease is associated with hippocampal atrophy. Mov Disord 2003, 18:784-790.

40. Pereira JB, Junqué C, Bartrés-Faz D, Ramírez-Ruiz B, Marti M-J, Tolosa E: Regional vulnerability of hippocampal subfields and memory deficits in Parkinson's disease. Hippocampus 2013, 23:720-728.

41. Zhang Y, Zhang J, Xu J, Wu X, Zhang Y, Feng H, Wang J, Jiang T: Cortical gyrification reductions and subcortical atrophy in Parkinson's disease. Mov Disord 2014, 29:122-126.

42. Deheshi S, Pasqualotto BA, Rintoul GL: Mitochondrial trafficking in neuropsychiatric diseases. Neurobiol Dis 2013, 51:66-71.

43. Chaturvedi RK, Flint Beal M: Mitochondrial diseases of the brain. Free Radic Biol Med 2013, 63:1-29.

44. Ridge PG, Koop A, Maxwell TJ, Bailey MH, Swerdlow RH, Kauwe JSK, Honea RA: Mitochondrial haplotypes associated with biomarkers for Alzheimer's disease. PLoS One 2013, 8:e74158.

45. Higgins LG, Hayes JD: Mechanisms of induction of cytosolic and microsomal glutathione transferase (GST) genes by xenobiotics and pro-inflammatory agents. Drug Metab Rev 2011, 43:92-137.

46. Jakobsson PJ, Mancini JA, Riendeau D, Ford-Hutchinson AW: Identification and characterization of a novel microsomal enzyme with glutathione-dependent transferase and peroxidase activities. J Biol Chem 1997, 272:22934-22939.

47. Amor S, Peferoen LAN, Vogel DYS, Breur M, van der Valk P, Baker D, van Noort JM: Inflammation in neurodegenerative diseases-an update. Immunology 2014, 142:151-166.

48. Schröder O, Sjöström M, Qiu H, Stein J, Jakobsson PJ, Haeggström JZ: Molecular and catalytic properties of three rat leukotriene $C(4)$ synthase homologs. Biochem Biophys Res Commun 2003, 312:271-276.

49. Schröder O, Sjöström M, Qiu H, Jakobsson P-J, Haeggström JZ: Microsomal glutathione S-transferases: selective up-regulation of leukotriene C4 synthase during lipopolysaccharide-induced pyresis. Cell Mol Life Sci 2005, 62:87-94.

50. Python; https://www.python.org

51. Novak NM, Stein JL, Medland SE, Hibar DP, Thompson PM, Toga AW: EnigmaVis: online interactive visualization of genome-wide association studies of the enhancing neurolmaging genetics through meta-analysis (ENIGMA) consortium. Twin Res Hum Genet 2012, 15:414-418.

52. MammalHom; http://depts.washington.edu/l2l/mammalhom.html.

53. Mouse Genome Informatics; http://www.informatics.jax.org/.

54. HomoloGene; http://www.ncbi.nlm.nih.gov/homologene.

55. R Core Team: R: A Language and Environment for Statistical Computing. Vienna, Austria: R Foundation for Statistical Computing; 2013.

56. Bacanu SA, Devlin B, Roeder K: The power of genomic control. Am J Hum Genet 2000, 66:1933-1944

57. Rosenblatt JD, Stein JL: RRHO: Test overlap using the Rank-Rank Hypergeometric test; 2013. R package version 1.0.0; http://www.bioconductor.org/packages/ release/bioc/html/RRHO.html.

58. Wang J, Williams RW, Manly KF: WebQTL: web-based complex trait analysis. Neuroinformatics 2003, 1:299-308.

59. Mulligan MK, Wang X, Adler AL, Mozhui K, Lu L, Williams RW: Complex control of $\mathrm{GABA}(\mathrm{A})$ receptor subunit $\mathrm{mRNA}$ expression: variation, covariation, and genetic regulation. PLoS One 2012, 7:e34586.

60. Huang DW, Sherman BT, Lempicki RA: Systematic and integrative analysis of large gene lists using DAVID bioinformatics resources. Nat Protoc 2008, 4:44-57.

61. Huang DW, Sherman BT, Lempicki RA: Bioinformatics enrichment tools: paths toward the comprehensive functional analysis of large gene lists. Nucleic Acids Res 2009, 37:1-13.

62. Ashburner M, Ball CA, Blake JA, Botstein D, Butler H, Cherry JM, Davis AP, Dolinski K, Dwight SS, Eppig JT, Harris MA, Hill DP, Issel-Tarver L, Kasarskis A, 
Lewis S, Matese JC, Richardson JE, Ringwald M, Rubin GM, Sherlock G: Gene ontology: tool for the unification of biology. The gene ontology consortium. Nat Genet 2000, 25:25-29.

63. Kanehisa M, Goto S: KEGG: kyoto encyclopedia of genes and genomes. Nucleic Acids Res 2000, 28:27-30.

64. Hunter $\mathrm{S}$, Jones $\mathrm{P}$, Mitchell A, Apweiler R, Attwood TK, Bateman A, Bernard T, Binns D, Bork P, Burge S, de Castro E, Coggill P, Corbett M, Das U, Daugherty L, Duquenne L, Finn RD, Fraser M, Gough J, Haft D, Hulo N, Kahn D, Kelly E, Letunic I, Lonsdale D, Lopez R, Madera M, Maslen J, McAnulla C, McDowall J, et al: InterPro in 2011: new developments in the family and domain prediction database. Nucleic Acids Res 2012, 40:D306-D312.

65. Edgar R, Domrachev M, Lash AE: Gene expression omnibus: NCBI gene expression and hybridization array data repository. Nucleic Acids Res 2002, 30:207-210

66. Barrett T, Wilhite SE, Ledoux P, Evangelista C, Kim IF, Tomashevsky M, Marshall KA, Phillippy KH, Sherman PM, Holko M, Yefanov A, Lee H, Zhang N, Robertson CL, Serova N, Davis S, Soboleva A: NCBI GEO: archive for functional genomics data sets-update. Nucleic Acids Res 2013, 41(Database issue):D991-D995.

67. Chesler EJ, Wang J, Lu L, Qu Y, Manly KF, Williams RW: Genetic correlates of gene expression in recombinant inbred strains: a relational model system to explore neurobehavioral phenotypes. Neuroinformatics 2003, 1:343-357.

68. Gray KA, Daugherty LC, Gordon SM, Seal RL, Wright MW, Bruford EA: Genenames.org: the HGNC resources in 2013. Nucleic Acids Res 2013, 41(Database):D545-D552

69. HGNC Database; http://www.genenames.org/.

doi:10.1186/1471-2164-15-850

Cite this article as: Ashbrook et al: Joint genetic analysis of

hippocampal size in mouse and human identifies a novel gene

linked to neurodegenerative disease. BMC Genomics 2014 15:850

\section{Submit your next manuscript to BioMed Central and take full advantage of:}

- Convenient online submission

- Thorough peer review

- No space constraints or color figure charges

- Immediate publication on acceptance

- Inclusion in PubMed, CAS, Scopus and Google Scholar

- Research which is freely available for redistribution 